\title{
Step coverage modeling of thin films in atomic layer deposition
}

\author{
Ja-Yong Kim, ${ }^{\text {a) }} \mathrm{Ji}-$ Hoon Ahn, and Sang-Won Kang ${ }^{\text {b) }}$ \\ Department of Materials Science and Engineering, Korea Advanced Institute of Science and Technology, \\ 373-1, Guseong-dong, Yuseong-gu, Daejeon 305-701, Korea \\ Jin-Hyock Kim \\ Hynix Semiconductor Incorporated, San 136-1, Ami-ri, Bubal-eub, Icheon-si, Kyoungki-do 467-701, Korea
}

(Received 5 November 2006; accepted 24 January 2007; published online 2 April 2007)

\begin{abstract}
A film growth model on microfeatures is proposed to evaluate step coverage depending on precursor injection time in atomic layer deposition. The proposed model is based on that the chemisorption rate of precursors at a certain position along the depth of a microfeature is determined by the total flux of precursors and the sticking probability. The total flux includes the flux coming from the entrance of a microfeature and the flux reflected from other positions inside a microfeature, and the sticking probability depends on the surface coverage of chemisorbed precursor, which is a function of precursor injection time. The proposed model was applied to the deposition of $\mathrm{Al}_{2} \mathrm{O}_{3}$ films on $0.3 \mu \mathrm{m}$ diameter holes with an aspect ratio of 10. It was confirmed that the experimental data for step coverage depending on precursor injection time follow the trend predicted by the proposed model. (C) 2007 American Institute of Physics. [DOI: 10.1063/1.2714685]
\end{abstract}

\section{INTRODUCTION}

As the minimum feature size of semiconductor devices decreases, the memory cell dimension also decreases. The required cell capacitance in dynamic random access memory (DRAM) has been made possible by three-dimensional cells with high aspect ratios. In particular, aspect ratios of more than 100 will be used in the near future in semiconductor memories. ${ }^{1}$ Therefore, good conformality is one of the most important requirements for fabricating microelectronic devices.

Atomic layer deposition (ALD) is a promising technique suitable for growing thin films with uniform thickness on microfeatures with high aspect ratios..$^{2-7}$ The key feature of ALD is surface-limited reactions between alternately dosed precursor and reactant gases. ${ }^{8-11}$ Under such conditions film growth is self-limiting, ensuring not only simple and accurate film thickness control but also excellent film step coverage. Furthermore, due to the alternate dosing of precursor and reactant gases, detrimental gas-phase reactions are inherently eliminated.

However, even in ALD, as aspect ratios increase severely, it is not trivial to obtain conformal film thickness on microfeatures. To support this task, it is needed to investigate theoretically the film deposition inside a microfeature in ALD and predict process time required to achieve reasonable film step coverage. The film thickness profile inside a microfeature depends on the chemisorption rate of precursors at each position along the depth of a microfeature. In order to achieve reasonable film step coverage on a microfeature, precursor injection time should be extended until the bottom of a microfeature is sufficiently covered with chemisorbed precursor. To evaluate the precursor injection time required to achieve reasonable film step coverage on a microfeature, it is

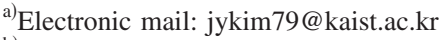

${ }^{b)}$ Electronic mail: swkang@kaist.ac.kr
}

necessary to understand the film deposition inside a microfeature in the nonsaturation region as well as the saturation region.

To date, only a few theoretical descriptions for estimating film step coverage in ALD have been reported in the literature. $^{12-15}$ Recently, a kinetic model for step coverage in ALD has been reported. ${ }^{12}$ In this model, it has been assumed that the sticking probability is unity, and the precursor injection time required to saturate up to the bottom inside a microfeature is evaluated. This model is useful in evaluating precursor injection time in the saturation region but is not easy to apply to film deposition in the nonsaturation region.

In this paper, a film growth model on microfeatures in ALD is proposed to understand more the film deposition inside a microfeature in the nonsaturation region as well as the saturation region. The proposed model was applied to the deposition of $\mathrm{Al}_{2} \mathrm{O}_{3}$ films on $0.3 \mu \mathrm{m}$ diameter holes with an aspect ratio of 10 . It was confirmed that the experimental data for step coverage depending on precursor injection time follow the trend predicted by the proposed model.

\section{MODEL DESCRIPTION}

\section{A. Mathematical derivation}

The geometry of a hole in this model is shown in Fig. 1(a). The cylindrical coordinate system is used and the origin of the coordinate system is fixed at the center of the bottom, as indicated in Fig. 1(b). Some of the assumptions in this model are listed here. (1) The entrance of a hole is exposed to an ideal gas with precursor concentration $n_{v}$ and a Maxwellian distribution of velocities. ${ }^{12,16}$ This model is focused on the feature scale and, thus, it is considered the case in which the flux from the reactor volume to the entrance of a hole is assumed to be constant in time. That is, it is assumed that there is negligible depletion of precursors from the gas just outside a hole because of sufficiently high concentration of precursors, fast gas-phase diffusion within the reactor vol- 


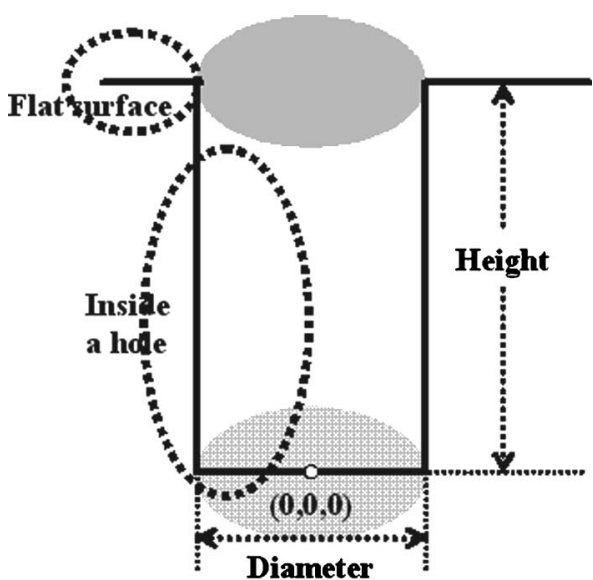

(a)

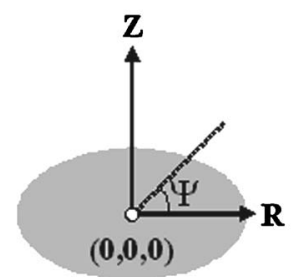

Cylindrical coor dinate system $(R, \Psi, Z)$

(b)

FIG. 1. (a) Schematic cross section of a hole and (b) cylindrical coordinate system.

ume, and rapid flow of precursors over the flat surface. (2) The precursors move by molecular flow inside a hole. ${ }^{12,16}$ This is a realistic assumption for submicrometer diameters and gas pressures around $1-5$ torr, since the mean free paths are much larger than the hole diameters. (3) The adsorption rate is much higher than the desorption rate, so the desorption rate is ignored. ${ }^{17}$ It implies that the interaction of the precursors with the surface is strong enough to give a very low desorption rate. If substrate temperature is not so high, this assumption would be valid. ${ }^{18}$ Generally, in ALD, this assumption follows quite directly from the self-limiting requirements that the adsorption must be irreversible, that is, the desorption rate is practically zero. ${ }^{19}$ (4) During the reactant pulse, all precursors chemisorbed on the surface are converted to the solid film.

\section{Mathematical derivation of flux}

Kinetic theory gives the impingement flux on the flat surface, as shown in Eq. (1), ${ }^{20}$

$$
J_{f}^{q}=\frac{n_{v} \bar{c}}{4},
$$

where $J_{f}^{q}$ is the flux of precursors that strikes the flat surface in the $q$ th cycle $\left(\mathrm{m}^{-2} \mathrm{~s}^{-1}\right), n_{v}$ is the precursor concentration near the entrance of a hole $\left(\mathrm{m}^{-3}\right)$, and $\bar{c}$ is the average velocity of precursors $\left(\mathrm{m} \mathrm{s}^{-1}\right)$.

The total flux at position $(r, \varphi, z)$ inside a hole includes the flux coming from the entrance of a hole and the flux reflected from other positions inside a hole. First, the flux at

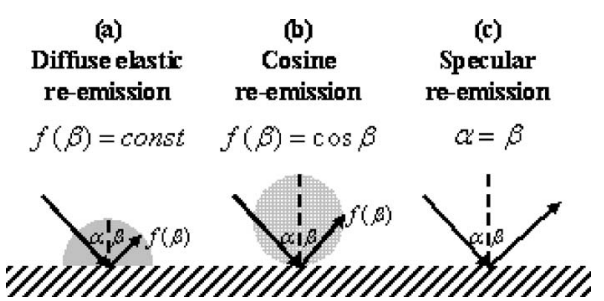

FIG. 2. Reemission mechanisms of incident molecules impinging on the surface: (a) diffuse elastic reemission, (b) cosine reemission, and (c) specular reemission.

position $(r, \varphi, z)$ inside a hole coming from the entrance of a hole is the sum of all flux coming from each position on the entrance of a hole. Then, the flux at position $(r, \varphi, z)$ inside a hole reflected from other positions inside a hole is determined by the flux reflected at other positions inside a hole and reemission mechanism. When the precursors collide with the surface, a portion of the precursors chemisorbs on the surface and the rest are reflected. The ratio of the precursors chemisorbed on the surface to the precursors colliding with the surface is termed the sticking probability $[S(\theta)]$. The probability that the precursors are reflected is $1-S(\theta)$; thus the flux reflected at the surface is less than the impingement flux by this factor. Also, various types of reemission have been studied by using specially treated substrates and molecular beams under high vacuum systems, and it was revealed that the reemission mechanism was affected by the structure of the surface and the surface contamination. ${ }^{21-23}$ Reemission mechanisms that were examined in these studies were diffuse elastic reemission, cosine reemission, and specular reemission, as indicated in Fig. 2. In diffuse elastic reemission and cosine reemission, there exists strong interaction between incoming molecules and the surface, so that molecules lose all information about previous incoming trajectory. In diffuse elastic reemission, molecules have equal probability of being reflected into any direction above the surface. In cosine reemission, the probability that molecules are reflected with angle $\beta$ is proportional to cosine function, i.e., $\cos \beta$, where $\beta$ is defined as the angle between the direction of reemission and the direction normal to the surface. In specular reemission, molecules experience negligible interaction with the surface so that the angle of reemission equals the incidence angle. Specular reemission has been observed in molecular beam experiments when a temperature of incoming molecules approaches that of the surface and the surface is atomically free of contamination. These experiments often use noble gas molecules and polished single crystal metals.

Therefore, the flux of precursors that strikes position $(r, \varphi, z)$ inside a hole on the second collision in the $q$ th cycle $\left[{ }^{2} J_{h(r, \varphi, z)}^{q}\right]$ is given by Eq. (2),

$$
{ }^{2} J_{h(r, \varphi, z)}^{q}=\int_{A_{H}}\left\{1-S\left(\theta_{h\left(r^{\prime}, \varphi^{\prime}, z^{\prime}\right)}^{q}\right)\right\}^{1} J_{h\left(r^{\prime}, \varphi^{\prime}, z^{\prime}\right)}^{q} f(s, \alpha, \beta) d A_{H},
$$

where ${ }^{1} J_{h(r, \varphi, z)}^{q}$ is the flux of precursors that strikes position $(r, \varphi, z)$ inside a hole on the first collision in the $q$ th cycle $\left(\mathrm{m}^{-2} \mathrm{~s}^{-1}\right), f(s, \alpha, \beta)$ is a function related to the reemission 
mechanism, each position inside a hole is connected by a line segment of length $s(\mathrm{~m})$, normal vectors at each position inside a hole form the angles of $\alpha$ and $\beta$ with respect to this connecting line, and $d A_{H}$ is the differential surface area inside a hole.

The flux of precursors that strikes position $(r, \varphi, z)$ inside a hole on the third collision in the $q$ th cycle $\left[{ }^{3} J_{h(r, \varphi, z)}^{q}\right]$ is given by Eq. (2), with the superscripts on the left incremented by 1 ,

$$
{ }^{3} J_{h(r, \varphi, z)}^{q}=\int_{A_{H}}\left\{1-S\left(\theta_{h\left(r^{\prime}, \varphi^{\prime}, z^{\prime}\right)}^{q}\right)\right\}^{2} J_{h\left(r^{\prime}, \varphi^{\prime}, z^{\prime}\right)}^{q} f(s, \alpha, \beta) d A_{H} .
$$

This accounting procedure can be continued. The total flux of precursors that strikes position $(r, \varphi, z)$ inside a hole in the $q$ th cycle $\left[J_{h(r, \varphi, z)}^{q}\right]$ is given by Eq. (4),

$$
J_{h(r, \varphi, z)}^{q}={ }^{1} J_{h(r, \varphi, z)}^{q}+{ }^{2} J_{h(r, \varphi, z)}^{q}+{ }^{3} J_{h(r, \varphi, z)}^{q}+\cdots
$$

\section{Mathematical derivation of surface coverage}

The surface coverage of chemisorbed precursor $(\theta)$ denotes the ratio of the surface area covered by the chemisorbed precursor to the exposed surface area,

$$
\theta=\frac{K}{K_{\max }}
$$

where $K$ is the number of chemisorbed precursor per unit area $\left(\mathrm{m}^{-2}\right)$ and $K_{\max }$ is the maximum number of chemisorbed precursor per unit area $\left(\mathrm{m}^{-2}\right)$.

The chemisorption rate of precursors $\left(R_{\mathrm{ch}}\right)$ is given by Eqs. (6) and (7). The chemisorption rate of precursors can be obtained by multiplying the total flux of precursors by the sticking probability,

$$
\begin{aligned}
& R_{\mathrm{ch}}=\frac{d K}{d t_{\text {p.t. }}}=K_{\max } \frac{d \theta}{d t_{\text {p.t. }}}, \\
& R_{\mathrm{ch}}=S(\theta) J_{f}^{q} \text { or } h(r, \varphi, z),
\end{aligned}
$$

where $t_{\text {p.t. }}$ is the precursor injection time $(\mathrm{s})$. The sticking probability depends on the surface coverage of chemisorbed precursor and usually decreases as the surface coverage of chemisorbed precursor increases. Langmuir suggested the following equation for the sticking probability as a function of the surface coverage, ${ }^{24}$

$$
S(\theta)=S(0)(1-\theta)^{n},
$$

where $S(0)$ is the initial sticking probability and $n$ is the adsorption order.

From Eqs. (6)-(8), the surface coverage of chemisorbed precursor at the flat surface or at position $(r, \varphi, z)$ inside a hole in the $q$ th cycle $\left[\theta_{f}^{q}\right.$ or $\left.h(r, \varphi, z)\right]$ is given by Eq. (9),
TABLE I. The summary of model parameters that should be extracted in order to apply the proposed model to the deposition of thin films.

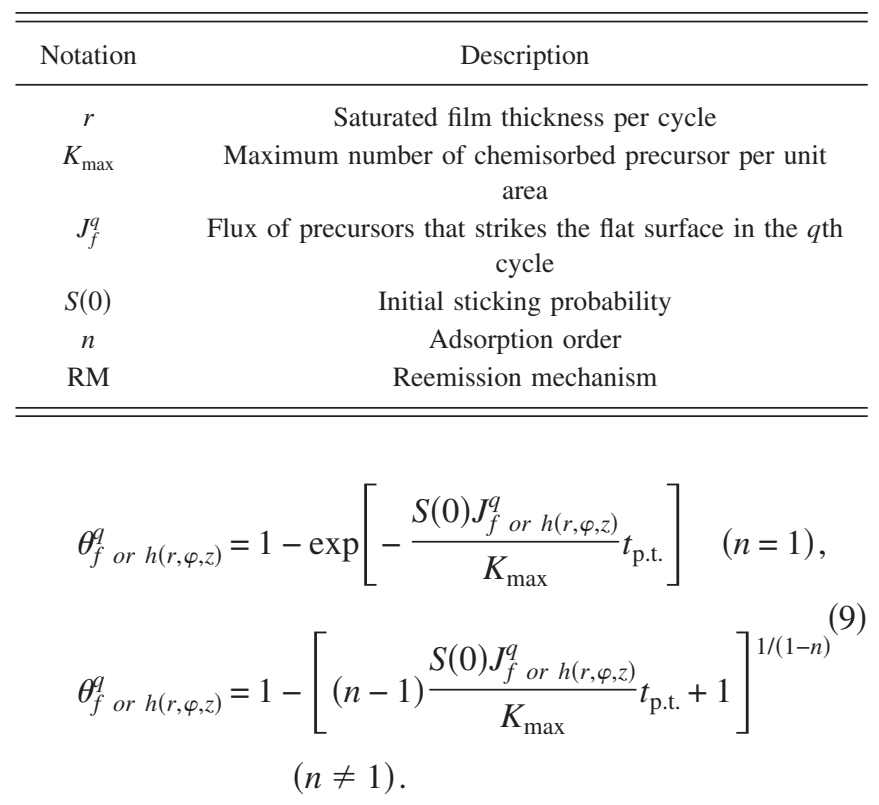

\section{Mathematical derivation of film thickness}

The film thickness newly formed from the chemisorbed precursor at the flat surface or at position $(r, \varphi, z)$ inside a hole in the $q$ th cycle $\left[T_{f}^{q}\right.$ or $\left.h(r, \varphi, z)\right]$ is given by Eq. (10),

$$
\begin{aligned}
T_{f \text { or } h(r, \varphi, z)=}^{q} \theta_{f \text { or } h(r, \varphi, z)}^{q} & \left\{1-\exp \left[-\frac{\left.\left.S(0) J_{f \text { or } h(r, \varphi, z)}^{q} t_{\text {p.t. }}\right]\right\} \quad(n=1),}{K_{\max }}=r \theta_{f}^{q} \text { or } h(r, \varphi, z)\right.\right. \\
T_{f \text { or } h(r, \varphi, z)}^{q} & \left\{1-\left[(n-1) \frac{\left.\left.\left.S(0) J_{f \text { or } h(r, \varphi, z)}^{q} t_{\text {p.t. }}+1\right]\right]_{\max }^{1 /(1-n)}\right\}}{(n \neq 1),}\right.\right.
\end{aligned}
$$

where $r$ is the saturated film thickness per cycle [in ML (monolayer)].

\section{B. Parameter extraction method}

The model parameters that should be extracted are summarized in Table I. These model parameters can be obtained by fitting the experimental data for film thickness per cycle at the flat surface and at the bottom inside a hole depending on precursor injection time to the proposed model.

Figure 3 shows the process of the model parameter extraction. At step 1, the saturated film thickness per cycle $(r)$ and the maximum number of chemisorbed precursor per unit area $\left(K_{\max }\right)$ are extracted from the experimental data for film thickness per cycle in the saturation region. $r$ is the maximum film thickness during one deposition cycle and $K_{\max }$ is obtained from Rutherford backscattering spectroscopy (RBS) analysis. 


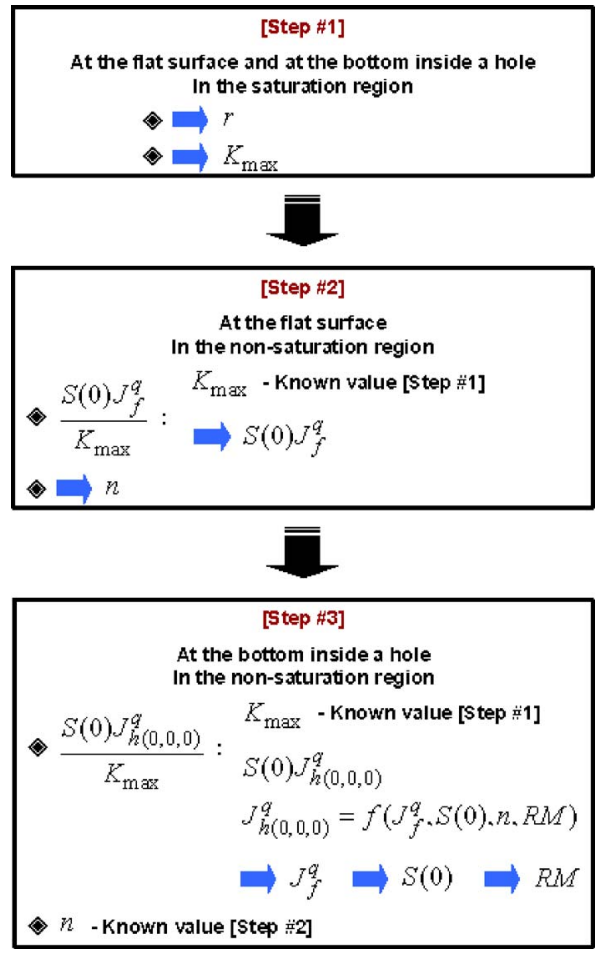

FIG. 3. (Color online) Process of the model parameter extraction.

$$
K_{\max }=r\left(\rho_{v}\right)^{2 / 3},
$$

where $\rho_{v}$ is the volume density of film $\left(\mathrm{m}^{-3}\right)$. As indicated in Eq. (10), the precursor injection time that is arrived at the maximum film thickness per cycle at the flat surface is determined by the adsorption order $(n)$ and the product of the flux of precursors that strikes the flat surface in the $q$ th cycle $\left(J_{f}^{q}\right)$ and the initial sticking probability $(S(0))$. Therefore, at step 2,n and the product of $J_{f}^{q}$ and $S(0)$ are obtained by fitting the experimental data for film thickness per cycle at the flat surface depending on precursor injection time to the proposed model. As indicated in Eq. (10), the precursor injection time that is arrived at the maximum film thickness per cycle at the bottom inside a hole is determined by $n$ and the product of $J_{h(0,0,0)}^{q}$ and $S(0)$. At this time, $J_{h(0,0,0)}^{q}$ is a function of $J_{f}^{q}, S(0), n$, and reemission mechanism. Therefore, at step $3, J_{f}^{q}, S(0)$, and reemission mechanism are obtained by fitting the experimental data for film thickness per cycle at the bottom inside a hole depending on precursor injection time to the proposed model.

\section{SIMULATION RESULTS}

The effect of the model parameters on film thickness profile inside a hole as well as film thickness per cycle at the flat surface and at the bottom inside a hole was confirmed by simulation when, in the case of cosine reemission, the hole diameter and height are fixed at 0.3 and $3 \mu \mathrm{m}$, respectively. Precursor injection time of $1 \mathrm{~s}$ is chosen, which is mostly less than the time required to achieve $100 \%$ step coverage.

The effect of $J_{f}^{q}$ on the film thickness profile inside a hole is shown in Fig. 4. The distance between the $y$ axis (hole height) and the profile line indicates film thickness at the sidewall inside a hole, and the distance between the $x$

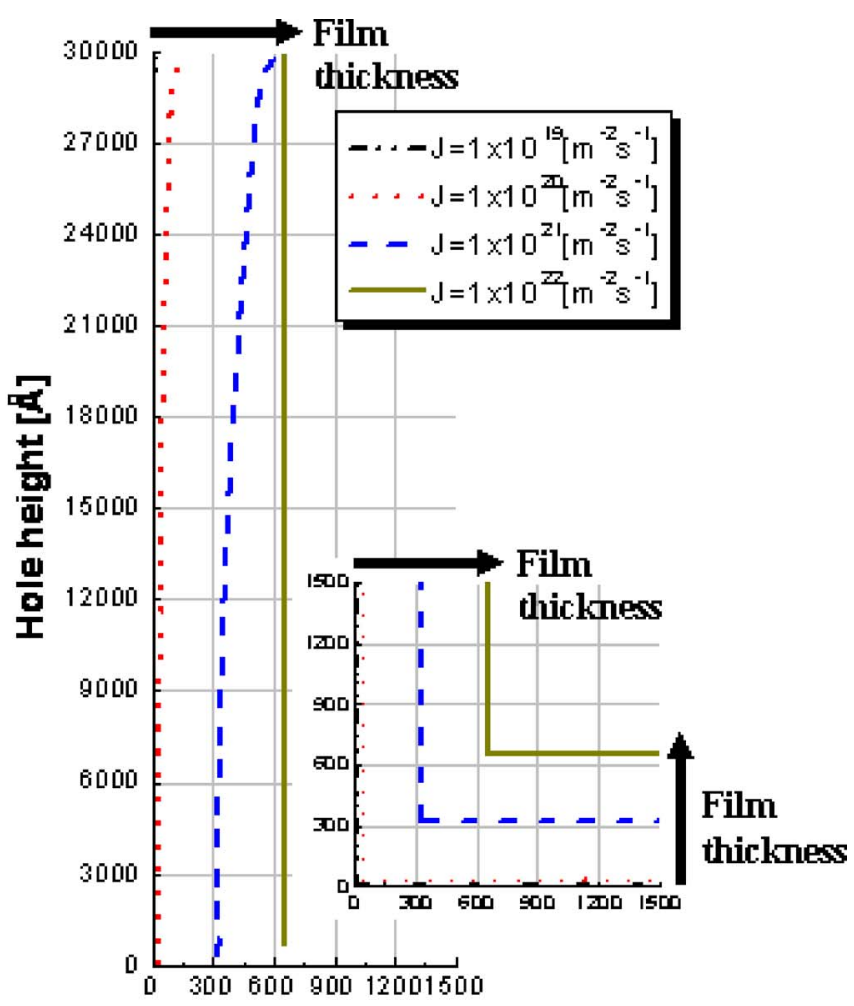

Hole radius [ $[\mathrm{A}]$

(a)

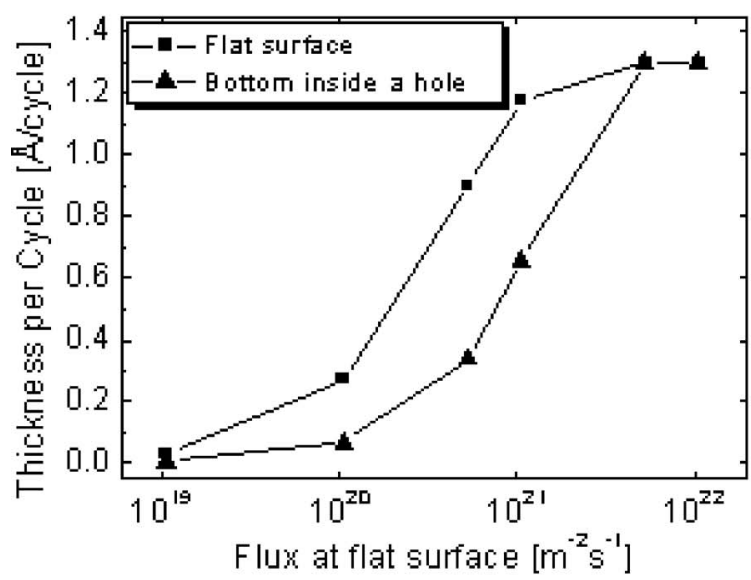

(b)

FIG. 4. (Color online) Simulated (a) film thickness profile inside a hole and (b) film thickness per cycle at the flat surface and at the bottom inside a hole as a function of $J_{f}^{q}$ at $S(0)=0.01, n=1$, and $t_{\text {p.t. }}=1 \mathrm{~s}$.

axis (hole radius) and the profile line indicates film thickness at the bottom inside a hole. $S(0)$ and $n$ are fixed at 0.01 and 1 , respectively. As $J_{f}^{q}$ increases, the film thickness inside a hole also increases because the number of precursor entering inside a hole per unit time increases.

The effect of $S(0)$ on the film thickness profile inside a hole is shown in Fig. 5. $J_{f}^{q}$ and $n$ are fixed at 1 $\times 10^{21} \mathrm{~m}^{-2} \mathrm{~s}^{-1}$ and 1 , respectively. The film thickness at a certain position along the depth of a hole is determined by the total flux of precursors and the sticking probability. When $S(0)$ is 1 , because most precursors are consumed at the upper portion of a hole and only a small number of precursors arrive at the lower portion of a hole, the film thickness differs 


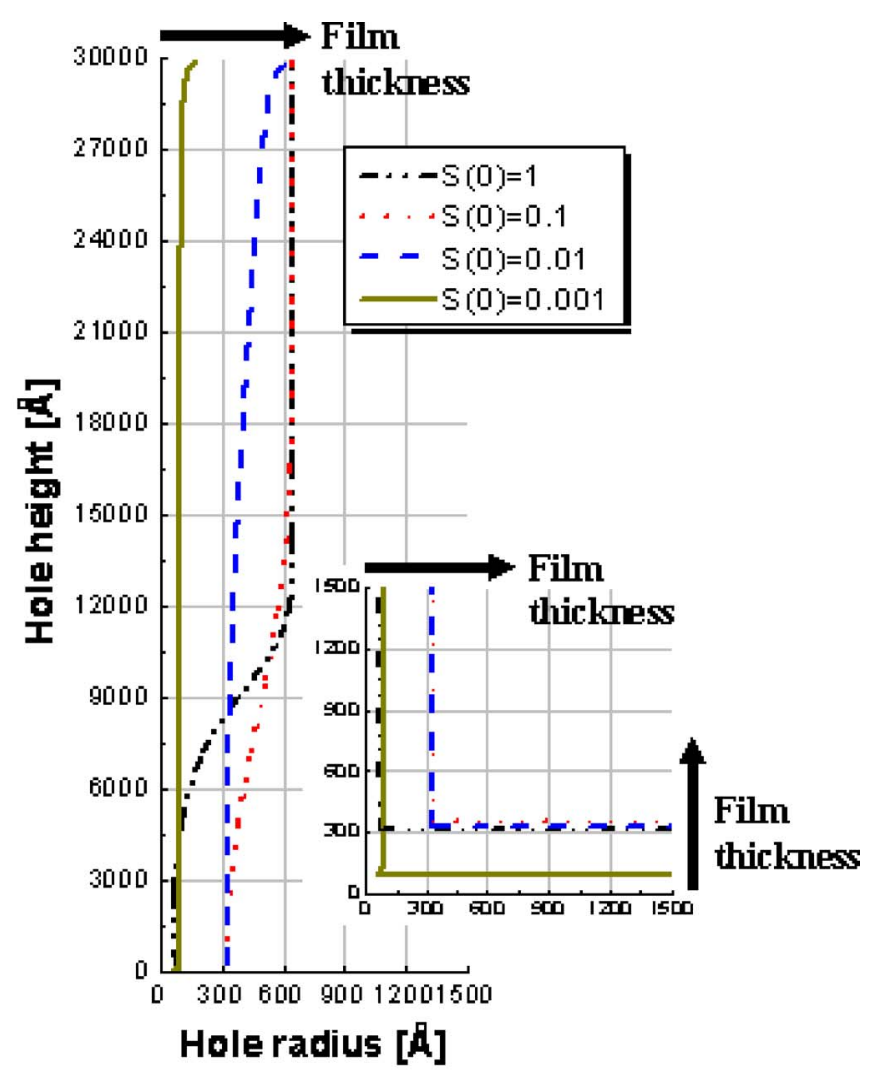

(a)

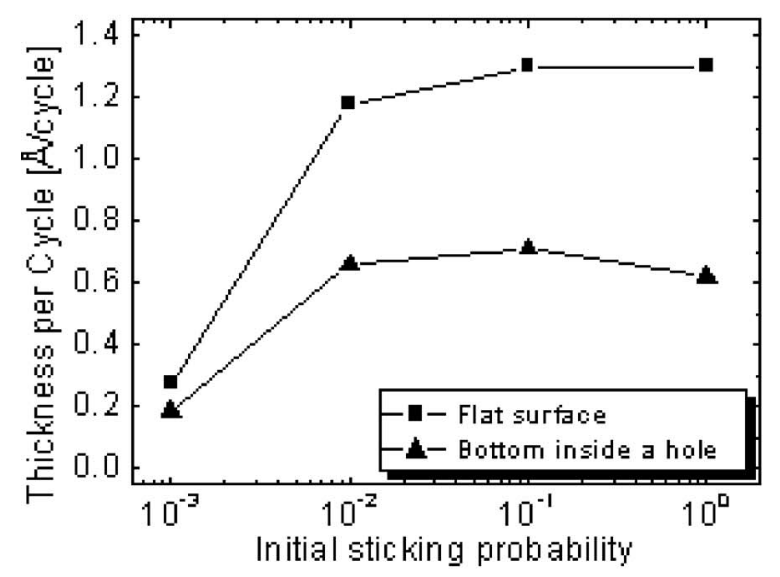

(b)

FIG. 5. (Color online) Simulated (a) film thickness profile inside a hole and (b) film thickness per cycle at the flat surface and at the bottom inside a hole as a function of $S(0)$ at $J_{f}^{q}=1 \times 10^{21} \mathrm{~m}^{-2} \mathrm{~s}^{-1}, n=1$, and $t_{\mathrm{p} . \mathrm{t}}=1 \mathrm{~s}$.

considerably from the upper portion to the lower portion of a hole. As $S(0)$ decreases, many more precursors arrive at the lower portion of a hole, so the difference in film thickness from the upper portion to the lower portion of a hole diminishes.

The effect of $n$ on the film thickness profile inside a hole is shown in Fig. 6. $J_{f}^{q}$ and $S(0)$ are fixed at $1 \times 10^{21} \mathrm{~m}^{-2} \mathrm{~s}^{-1}$ and 0.01 , respectively. As $n$ increases, the film thickness at the flat surface as well as at all positions inside a hole decreases. The sticking probability is proportional to $(1-\theta)^{n}$. Hence, as the surface coverage of chemisorbed precursor decreases, the effect of $n$ on the sticking probability decreases.

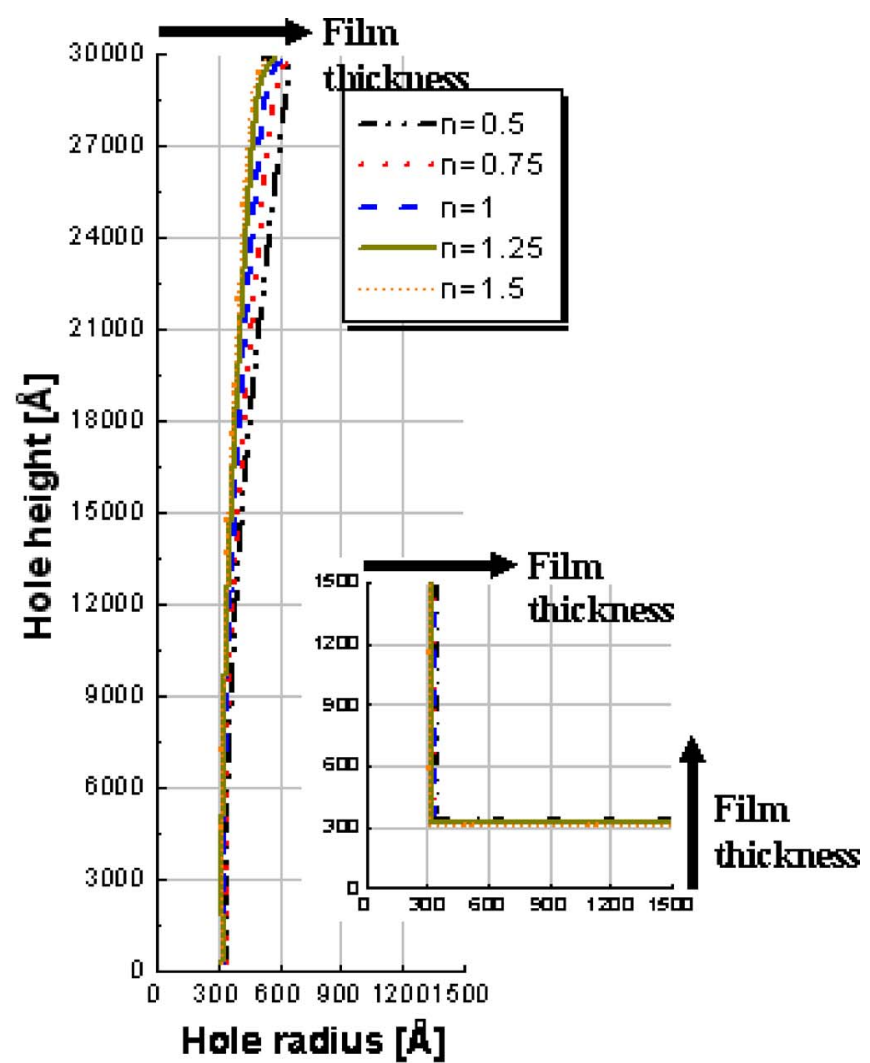

(a)

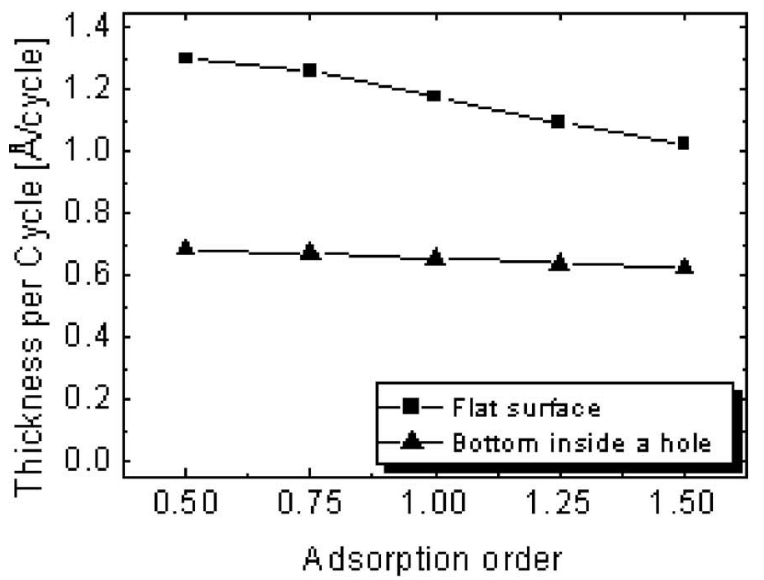

(b)

FIG. 6. (Color online) Simulated (a) film thickness profile inside a hole and (b) film thickness per cycle at the flat surface and at the bottom inside a hole as a function of $n$ at $J_{f}^{q}=1 \times 10^{21} \mathrm{~m}^{-2} \mathrm{~s}^{-1}, S(0)=0.01$, and $t_{\mathrm{p.t} .}=1 \mathrm{~s}$.

Therefore, as $n$ increases, variation of film thickness at all positions inside a hole is less than that at the flat surface.

\section{APPLICATION OF THE PROPOSED MODEL TO THE $\mathrm{Al}_{2} \mathrm{O}_{3}$ SYSTEM}

The proposed model was applied to the deposition of $\mathrm{Al}_{2} \mathrm{O}_{3}$ films using trimethylaluminum (TMA) as an Al precursor and $\mathrm{O}_{2}$ plasma as an oxidant on $0.3 \mu \mathrm{m}$ diameter holes with an aspect ratio of 10 at a substrate temperature of $225^{\circ} \mathrm{C}$ and a deposition pressure of 3 torr. The volume of the reactor is $97 \mathrm{~cm}^{3}$ (area of substrate holder, $324 \mathrm{~cm}^{2}$; distance between wafer and electrode, $0.3 \mathrm{~cm})$. TMA is a liquid 


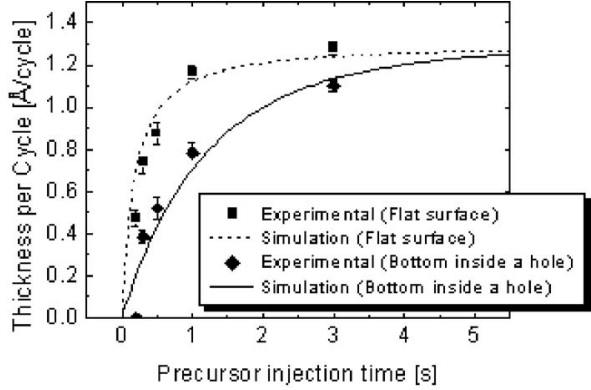

(a)

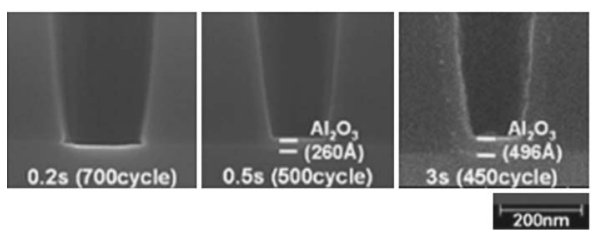

(b)

FIG. 7. (a) Dependence of $\mathrm{Al}_{2} \mathrm{O}_{3}$ film thickness per cycle at the flat surface and at the bottom inside a hole on TMA injection time at a TMA partial pressure of 0.01 torr and (b) cross sectional SEM images of $\mathrm{Al}_{2} \mathrm{O}_{3}$ film at the bottom inside a hole.

precursor with relatively high vapor pressures of 15.1 and 16.8 torr at 30 and $32{ }^{\circ} \mathrm{C}$, respectively. TMA, sustained at 30 and $32{ }^{\circ} \mathrm{C}$, was delivered to the reactor with $\mathrm{Ar}$ carrier gas at a flow rate of 50 SCCM (SCCM denotes cubic centimeter per minute at STP). The values of TMA partial pressures were about 0.01 and 0.04 torr. One deposition cycle of $\mathrm{Al}_{2} \mathrm{O}_{3}$ was composed of a TMA vapor pulse, a purge pulse for $3 \mathrm{~s}$, a pulse for an exposure to $\mathrm{O}_{2}$ plasma for $2 \mathrm{~s}$, and another purge pulse for $1 \mathrm{~s}$. The flow rates of purge $\mathrm{Ar}$ and $\mathrm{O}_{2}$ were 250 and $100 \mathrm{SCCM}$, respectively. The plasma power was kept at $150 \mathrm{~W}$. When a large enough dose of TMA was supplied, $\mathrm{Al}_{2} \mathrm{O}_{3}$ film thickness per cycle at the flat surface and at the bottom inside a hole was saturated at $1.3 \AA /$ cycle at the pulse time of $\mathrm{O}_{2}$ plasma of $1 \mathrm{~s}$. This implies that conversion of all precursors chemisorbed on the surface into the solid film was achieved. To induce the complete reaction between the precursors chemisorbed on the surface and the plasma, the enough pulse time of $\mathrm{O}_{2}$ plasma was given by setting at $2 \mathrm{~s}$. The film thickness was measured by field emission scanning electron microscopy (FESEM).

In order to apply the proposed model to the deposition of $\mathrm{Al}_{2} \mathrm{O}_{3}$ films, the model parameters, as indicated in Table I, should be extracted. Figure 7 shows (a) $\mathrm{Al}_{2} \mathrm{O}_{3}$ film thickness per cycle at the flat surface and at the bottom inside a hole depending on TMA injection time at a TMA partial pressure of 0.01 torr and (b) cross sectional SEM images of $\mathrm{Al}_{2} \mathrm{O}_{3}$ film at the bottom inside a hole. TMA injection time is varied from 0.2 to $3 \mathrm{~s}$, and the pulse time of $\mathrm{O}_{2}$ plasma is fixed at $2 \mathrm{~s} . \quad \mathrm{Al}_{2} \mathrm{O}_{3}$ film thickness per cycle is saturated at $1.3 \AA /$ cycle, and the monolayer thickness of $\mathrm{Al}_{2} \mathrm{O}_{3}$ film is $3.1 \AA .^{25,26}$ Thus, $r$ is 0.419 ML. From Eq. (11), $K_{\max }$ is $4.473 \times 10^{18} \mathrm{~m}^{-2} . n$ and the product of $J_{f}^{q}$ and $S(0)$ are obtained by fitting the experimental data at the flat surface to the proposed model. These are 1.9 and 2.505 $\times 10^{19} \mathrm{~m}^{-2} \mathrm{~s}^{-1}$, respectively. Also, each value of $J_{f}^{q}$ and $S(0)$ as well as the reemission mechanism is obtained by fitting the experimental data at the bottom inside a hole to the pro-

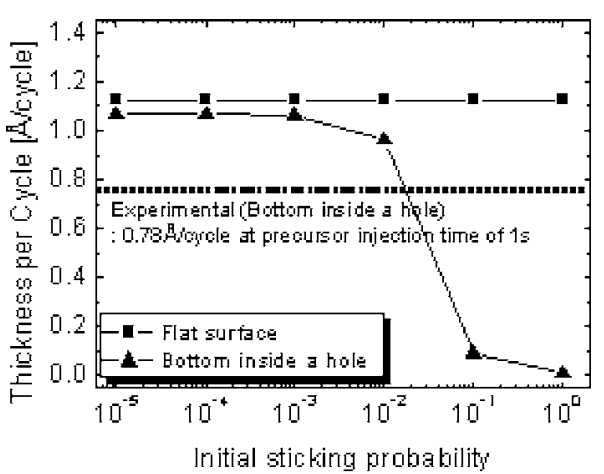

(a)

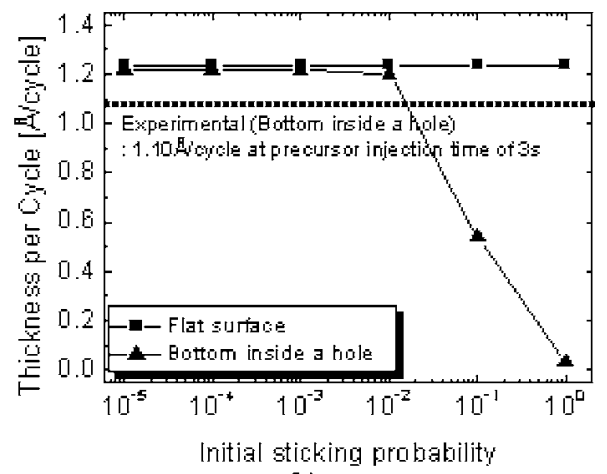

(b)

FIG. 8. Simulated film thickness per cycle at the flat surface and at the bottom inside a hole as a function of $S(0)$ for cosine reemission at (a) $t_{\text {p.t. }}$. $=1 \mathrm{~s}$ and (b) $t_{\text {p.t. }}=3 \mathrm{~s} \quad\left[r=0.419\right.$ ML, $K_{\max }=4.473 \times 10^{18} \mathrm{~m}^{-2}, \quad J_{f}^{q} S(0)$ $=2.505 \times 10^{19} \mathrm{~m}^{-2} \mathrm{~s}^{-1}$, and $\left.n=1.9\right]$.

posed model. Figure 8 shows simulated film thickness per cycle at the flat surface and at the bottom inside a hole as a function of $S(0)$ for cosine reemission at precursor injection times of (a) $1 \mathrm{~s}$ and (b) $3 \mathrm{~s}$ in the case that the product of $J_{f}^{q}$ and $S(0)$ is constant as $2.505 \times 10^{19} \mathrm{~m}^{-2} \mathrm{~s}^{-1}$. When precursor injection times are 1 and $3 \mathrm{~s}$, experimental film thicknesses per cycle at the bottom inside a hole are 0.78 and $1.10 \AA /$ cycle, respectively. As indicated in Fig. 8, in the case of cosine reemission, when $S(0)$ is $0.026\left(J_{f}^{q}\right.$ is 9.635 $\times 10^{20} \mathrm{~m}^{-2} \mathrm{~s}^{-1}$ ), a point of intersection between simulation and experimental results exists. However, in the case of diffuse elastic reemission, the model parameter extraction is impossible because a point of intersection between simulation and experimental results does not exist. Also, it has been known that specular reemission occurs for noble gas molecules and polished single crystal metals when a temperature of incoming molecules is the same as that of the surface but it could be varied by the surface contamination. ${ }^{21}$ In many cases, molecules experience non-negligible interaction with the surface before they reemit into free space, for example, when the surface is not atomically free of contamination. ${ }^{27-29}$ Thus, specular reemission is ruled out in the model parameter extraction. Therefore, $J_{f}^{q}, S(0)$, and reemission mechanism are obtained by fitting the experimental data at the bottom inside a hole to the proposed model, and these are $9.635 \times 10^{20} \mathrm{~m}^{-2} \mathrm{~s}^{-1}, 0.026$, and cosine reemission, respectively. Because of assumption (1), the model parameters obtained by fitting the experimental data for film thickness per 


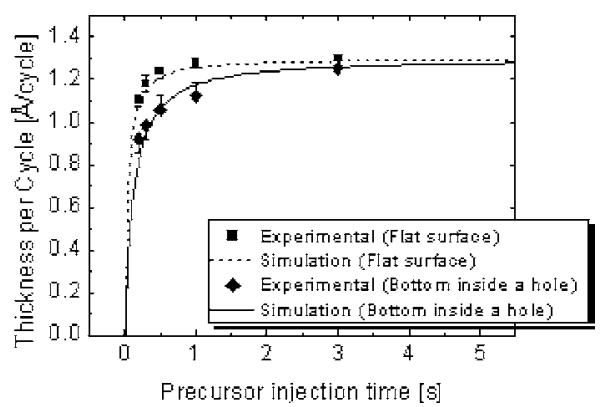

FIG. 9. Dependence of $\mathrm{Al}_{2} \mathrm{O}_{3}$ film thickness per cycle at the flat surface and at the bottom inside a hole on TMA injection time at a TMA partial pressure of 0.04 torr.

cycle at the flat surface and at the bottom inside a hole, depending on precursor injection time to the proposed model, may contain systematic error.

The effect of a TMA partial pressure on step coverage was confirmed. It is regarded that $J_{f}^{q}$ is a function of a TMA partial pressure. Figure 9 shows $\mathrm{Al}_{2} \mathrm{O}_{3}$ film thickness per cycle at the flat surface and at the bottom inside a hole depending on TMA injection time at a TMA partial pressure of 0.04 torr. By fitting the experimental data at the flat surface to the proposed model, the product of $J_{f}^{q}$ and $S(0)$ is obtained as $1.002 \times 10^{20} \mathrm{~m}^{-2} \mathrm{~s}^{-1}$. Because all experimental conditions were the same as the experimental conditions for extracting the model parameters with the exception of a TMA partial pressure, $S(0)$ is 0.026 . Thus, $J_{f}^{q}$ is obtained as 3.854 $\times 10^{21} \mathrm{~m}^{-2} \mathrm{~s}^{-1}$. The $\mathrm{Al}_{2} \mathrm{O}_{3}$ film thickness per cycle at the bottom inside a hole depending on TMA injection time was predicted by the proposed model under these simulation conditions, as indicated in Fig. 9. These expected simulation results at the bottom inside a hole well agreed with the experimental results.

In order to confirm the applicability of the proposed model, the predictions of the proposed model compare to those of earlier published model. ${ }^{12}$ In the proposed model, in the case of the deposition of $\mathrm{Al}_{2} \mathrm{O}_{3}$ films, the precursor injection time required to achieve $99 \%$ saturation at the flat surface and at position $(r, \varphi, z)$ inside a hole is given by $2654\left(K_{\max } / J_{f}^{q}\right)$ and $2654\left(K_{\max } / J_{h(r, \varphi, z)}^{q}\right)$. In earlier published model, when the aspect ratio of a hole is 15 , which is the final aspect ratio of the deposited hole at the end of the deposition at our application example, the precursor injection time required to achieve saturation at the flat surface and inside a hole is given by $K_{\max } / J_{f}^{q}$ and $410\left(K_{\max } / J_{f}^{q}\right)$. The precursor injection time required to achieve saturation in the proposed model is longer than that in earlier published model. The reason is thought that, in earlier published model, although any real adsorption will have less than $100 \%$ sticking probability, the calculations assumed a 100\% sticking probability.

\section{CONCLUSIONS}

A film growth model on microfeatures was proposed to evaluate step coverage depending on precursor injection time in ALD. The effect of the model parameters on film thickness profile inside a hole was confirmed by simulation. The proposed model was applied to the deposition of $\mathrm{Al}_{2} \mathrm{O}_{3}$ films on $0.3 \mu \mathrm{m}$ diameter holes with an aspect ratio of 10 . The model parameters were extracted by fitting the experimental data for $\mathrm{Al}_{2} \mathrm{O}_{3}$ film thickness per cycle at the flat surface and at the bottom inside a hole depending on TMA injection time to the proposed model. Also, it was confirmed that the experimental data for step coverage depending on TMA injection time were in good agreement with the model predictions at different partial pressures of TMA. This study should be helpful in making DRAM capacitors for semiconductor memories as well as in other applications requiring deposition inside microfeatures with high aspect ratios.

\section{ACKNOWLEDGMENT}

This work was supported by the Korea Research Foundation Grant funded by the Korean Government (MOEHRD) (KRF-2005-005-J09702).

\footnotetext{
${ }^{1}$ International Technology Roadmap for Semiconductors, 2005.

${ }^{2}$ M. Ritala, P. Kalsi, D. Riihela, K. Kukli, M. Leskela, and J. Jokinen, Chem. Mater. 11, 1712 (1999).

${ }^{3}$ J. S. Min, H. S. Park, and S. W. Kang, Appl. Phys. Lett. 75, 1521 (1999).

${ }^{4}$ M. Ritala, M. Leskela, J. P. Dekker, C. Mutsaers, P. J. Soininen, and J.

Skarp, Chem. Vap. Deposition 5, 7 (1999).

${ }^{5}$ J. E. Crowell, J. Vac. Sci. Technol. A 21, S88 (2003).

${ }^{6}$ H. Kim, J. Vac. Sci. Technol. B 21, 2231 (2003).

${ }^{7}$ O. Sneh, R. B. Clark-Phelps, A. R. Londergan, J. Winkler, and T. E. Seidel, Thin Solid Films 402, 248 (2002).

${ }^{8}$ M. Leskela and M. Ritala, J. Phys. IV 5, C5 (1995).

${ }^{9}$ M. Ritala, Appl. Surf. Sci. 112, 223 (1997).

${ }^{10}$ L. Niinisto, M. Ritala, and M. Leskela, Mater. Sci. Eng., B 41, 23 (1996).

${ }^{11}$ T. Suntola, Thin Solid Films 216, 84 (1992).

${ }^{12}$ R. G. Gordon, D. Hausmann, E. Kim, and J. Shepard, Chem. Vap. Deposition 9, 73 (2003).

${ }^{13}$ U. Schroeder, M. Gutsche, T. Hecht, A. Kersch, G. Prechtl, T. Popp, and H. Seidl, AVS Topical Conference on Atomic Layer Deposition 2002, Hanyang University, Seoul, Korea, 19-21 August 2002.

${ }^{14}$ M. K. Gobbert, V. Prasad, and T. S. Cale, J. Vac. Sci. Technol. B 20, 1031 (2002).

${ }^{15}$ J. W. Elam, D. Routkevitch, P. P. Mardilovich, and S. M. Goerge, Chem. Mater. 15, 3507 (2003).

${ }^{16}$ T. S. Cale, G. B. Raupp, and T. H. Gandy, J. Appl. Phys. 68, 3645 (1990).

${ }^{17}$ S. Sugahara, T. Kitamura, S. Imai, and M. Matsumura, Appl. Surf. Sci. 82/83, 380 (1994).

${ }^{18}$ Y. Sakuma, S. Muto, K. Nakajima, and N. Yokoyama, Appl. Surf. Sci. 82/83, 239 (1994)

${ }^{19}$ R. L. Puurunen, J. Appl. Phys. 97, 121301 (2005).

${ }^{20} \mathrm{G}$. N. Patterson, Introduction to the Kinetic Theory of Gas Flows (University of Toronto Press, Toronto, 1971).

${ }^{21}$ L. A. West and G. A. Somorjai, J. Chem. Phys. 54, 2864 (1971).

${ }^{22}$ S. Tomoda and K. Kodera, Surf. Sci. 45, 657 (1974).

${ }^{23}$ R. D. Levin and R. B. Bernstein, Molecular Reaction Dynamics and Chemical Reactivity (University of Oxford Press, New York, 1987).

${ }^{24}$ R. I. Masel, Principle of Adsorption and Reaction on Solid Surfaces (Wiley, New York, 1996).

${ }^{25}$ R. L. Puurunen, Chem. Vap. Deposition 9, 249 (2003).

${ }^{26}$ J. H. Kim, J. Y. Kim, and S. W. Kang, J. Appl. Phys. 97, 093505 (2005).

${ }^{27}$ H. C. Wulu, K. C. Saraswat, and J. P. McVittie, J. Electrochem. Soc. 138, 1831 (1991).

${ }^{28}$ J. B. Taylor, Phys. Rev. 35, 375 (1930).

${ }^{29}$ A. Ellett and V. W. Cohen, Phys. Rev. 52, 509 (1937).
} 